\title{
Chronic tophaceous gout in patients with psoriasis ${ }^{*}$
}

\author{
Laís Cruz Lobato ${ }^{1}$ \\ Maria Zeli Moreira Frota ${ }^{2}$ \\ Mônica Santos ${ }^{4,5}$
}

\author{
Jéssica Castiel Coutinho ${ }^{1}$ \\ Antonio Pedro Mendes Schettini ${ }^{3}$
}

DOI: http:/ / dx.doi.org/10.1590/abd1806-4841.20174895

\begin{abstract}
Psoriasis is a chronic inflammatory disease of multifactorial etiology influenced by genetic, immunological, and environmental factors. We report the case of a patient with psoriasis for more than 25 years who developed hyperuricemia and chronic tophaceous gout with unusual appearance. In psoriasis, hyperuricemia may occur by increased epidermal cell turnover, which accelerates purine metabolism and has uric acid as the product of its catabolism. The association of psoriasis with hyperuricemia can trigger the onset of gouty arthritis, and pose a greater risk of developing other inflammatory comorbidities. Therefore, it is important to periodically investigate uric acid levels in order to treat changes triggered by hyperuricemia. Keywords: Gout; Psoriasis; Uric acid
\end{abstract}

\section{INTRODUCTION}

Psoriasis is a chronic inflammatory disease that can affect the skin, joints, nails, and scalp. It has a multifactorial etiology, with the participation of genetic, immunological, and environmental factors. Several studies have demonstrated the role of inflammatory cells and mediators in its pathogenesis. It is defined as a systemic and immune-mediated inflammatory disease, which can be associated with other inflammatory diseases. ${ }^{1,2}$ One of these diseases is gout, a disorder caused by the deposition of urate crystals in joints and periarticular tissues. Its basic feature is hyperuricemia. It is defined by serum uric acid levels above $6.8 \mathrm{mg} / \mathrm{dl}(404 \mathrm{nmol} / \mathrm{L})$, which is the approximate saturation point of urate solubility under physiological conditions of temperature and $\mathrm{pH} .{ }^{3}$ Uric acid levels beyond blood solubility may form crystals, and consequently, gouty arthritis. This is a potentially destructive disease that not only deposits crystals within the joints and skin, but can also stimulate inflammatory cytokines and chemotactic factors for neutrophils. ${ }^{4}$ We report the case of a patient with psoriasis for more than 25 years who developed hyperuricemia and unsightly gouty tophus formation.

\section{CASE REPORT}

We report a 47-year-old male patient who had psoriasis since he was 19. In 2013, he also reported pain and edema in the right knee joints and right elbow, interpreted as psoriatic arthritis, for which he received classical systemic treatment with methotrexate. The cutaneous and joint manifestations evolved with partial improvement, and the patient stopped treatment for two years. He returned to dermatologic consultation with small erythematous scaly papules on the upper and lower limbs, and erythema and flaky scalp. However, his main complaint at that time was pain, volume increase, and severely swollen softened lesion on the side of the left foot, with a 6-month period evolution (Figure 1). Based on the clinical picture, we suspected of mycetoma, phaeohyphomycosis, atypical mycobacteriosis, septic arthritis, and gout tophaceous. The following tests were performed: complete blood count, blood glucose dosage, lipid profile, foot radiography, renal ultrasound, and skin biopsy. The material was sent to histopathological and mycological examinations. Seven days after biopsy, the lesion evolved with fistula formation and drainage of yellow-white exudate and lumpy material (Figure 2). Histological examination with hematox-

Received on 01.07.2015

Approved by the Advisory Board and accepted for publication on 13.01.2016

* Work performed at Fundação Alfredo da Matta (UAM) - Manaus (AM), Brazil.

Financial Support: None.

Conflict of Interest: None.

Private Clinic - Manaus (AM), Brazil.

Department of Mycology - School of Pharmaceutical Sciences - Universidade Federal do Amazonas (UFAM) - Manaus (AM), Brazil.

Department of Dermatopathology - Fundação Alfredo da Matta (FUAM) - Manaus (AM), Brazil.

Department of Psoriasis - Fundação Alfredo da Matta (FUAM) - Manaus (AM), Brazil.

School of Medicine - Universidade do Estado do Amazonas (UEA) - Manaus (AM), Brazil.

(C2017 by Anais Brasileiros de Dermatologia 
ylin-eosin showed hyperkeratosis and acanthosis in the epidermis. The dermis revealed thickened collagen with infiltration of mononuclear cells around the vessels and the presence of amorphous material, with a lightly eosinophilic appearance, surrounded by coarse bands of collagen tissue (Figure 3). Staining with periodic acid-Schiff (PAS) showed no fungal structures. The direct examination of the lumpy material sent to medical mycology laboratory showed the presence of spiculated structures compatible with crystal deposits (Figure 4). There was no fungal growth in the culture medium. Serum levels were the following: uric acid $(10.8 \mathrm{mg} / \mathrm{dl})$; glucose (119 mg/dL); total cholesterol (256 mg/dL); and HDL (36 mg/dL). The concentration of uric acid in the 24-hour urine specimen was $1.450 \mathrm{mg} / \mathrm{dL}$ (reference value up to $750 \mathrm{mg} / \mathrm{dL}$ ). No renal calculi were detected in kidney ultrasound. Left foot radiograph (dorsoplantar view) showed erosion and sclerosis of the forefoot bones, with preservation of joint spaces and bone density. Based on clinical and laboratory findings, the diagnosis of chronic tophaceous gout was confirmed. The patient was then sent to a concomitant rheumatologist follow-up, which started treatment with colchicine $(0.5$ mg every 12 hours orally). Allopurinol (300 mg orally) was added to the treatment 14 days later. Two months after the introduction

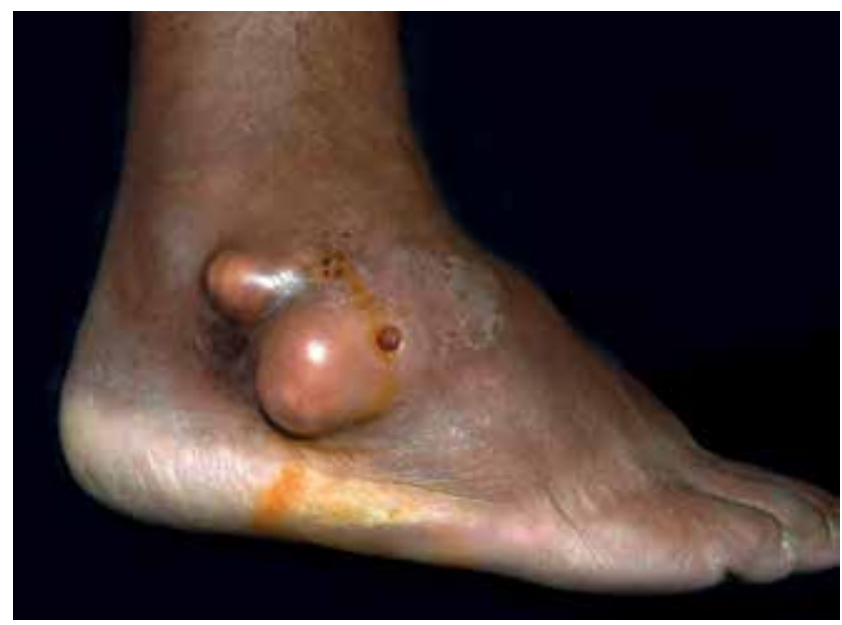

Figure 1: Severely swollen and softened lesion on the side of the left foot with a 6-month period evolution

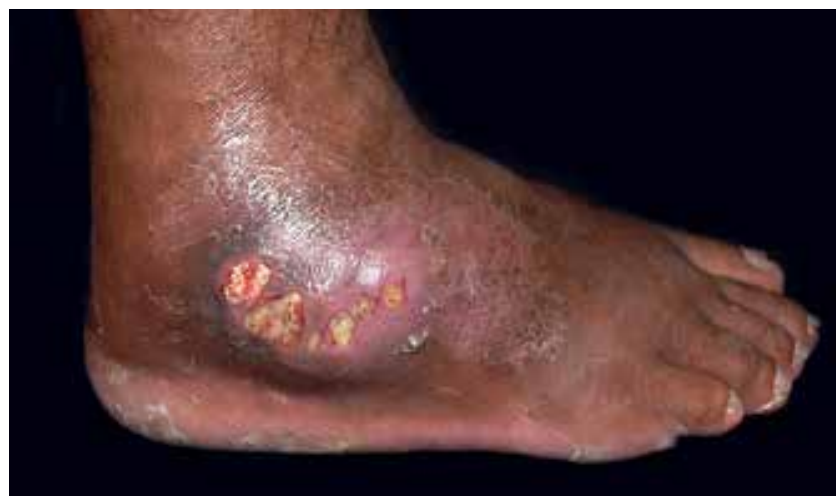

FIGURE 2: Seven days after biopsy, the lesion evolved with fistula formation and drainage of yellow-white exudate and lumpy material
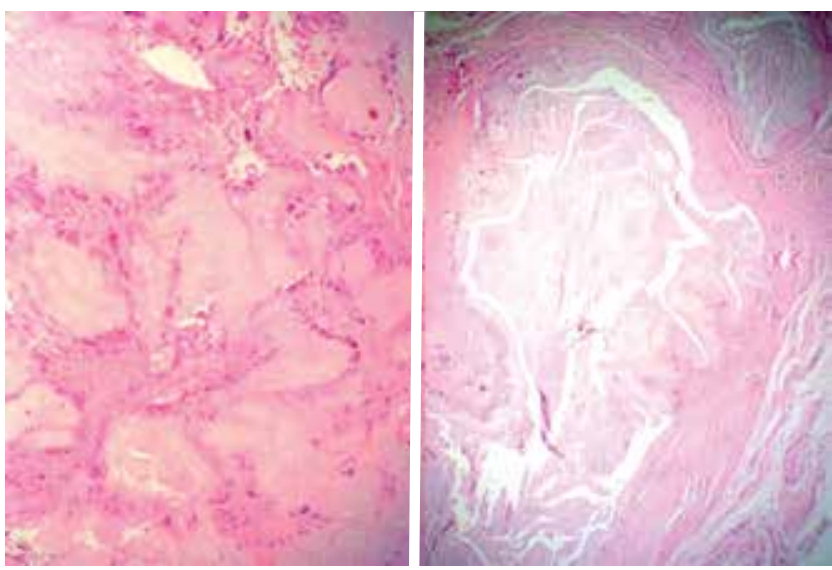

FIGURE 3: Histological examination with hematoxylin-eosin showed hyperkeratosis and acanthosis in the epidermis. The dermis revealed thickened collagen with infiltration of mononuclear cells around the vessels and the presence of amorphous material, with a lightly eosinophilic appearance, surrounded by coarse bands of collagen tissue

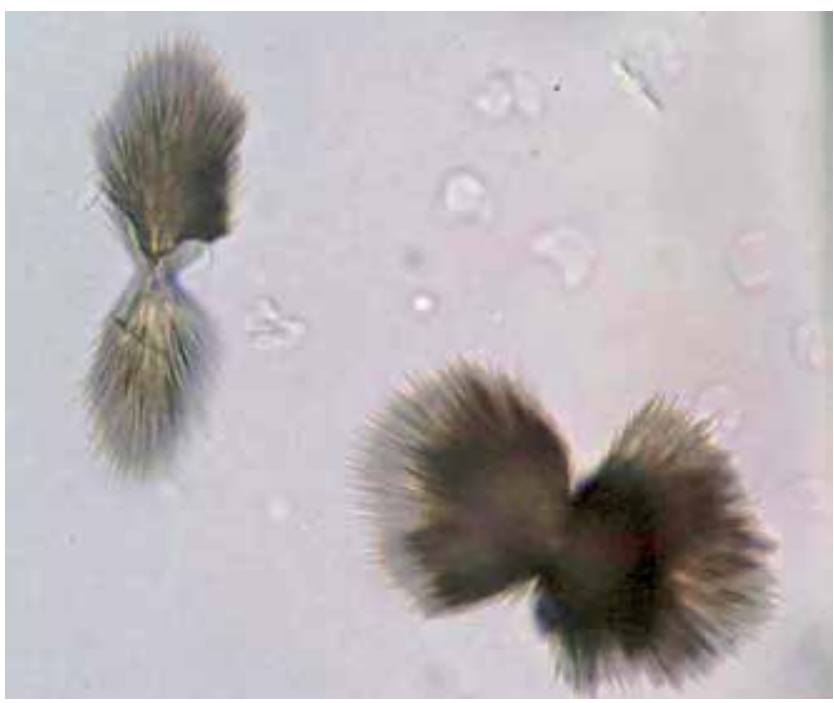

FIGURE 4: The direct examination of the lumpy material sent to medical mycology laboratory showed the presence of spiculated structures compatible with crystal deposits

of medications, we observed a normalization of uric acid concentration and a significant reduction of the gouty tophus lesion and pain. The patient has been followed at dermatology and reumatology clinical setting.

\section{DISCUSSION}

In psoriasis, hyperuricemia may occur by increased epidermal cell turnover, which accelerates purine metabolism and has uric acid as the product of its catabolism. Other factors also contribute to this hyperuricemia, such as alcohol consumption - especially fermented drinks - genetic predisposition, and metabolic syndrome coexistence. In our case, the patient used alcohol regularly and had a diagnosis of metabolic syndrome. Differential diagnosis of gouty 
arthritis include septic psoriatic, and rheumatoid arthritis. ${ }^{5}$ In the reported case, the involvement of soft tissue with edema and elimination of lumpy material raised the suspicion of mycetoma and mycobacteriosis. In the acute phase of chronic tophaceous gout, conventional radiography may reveal swelling of soft tissue and joint effusion. In the chronic phase, intra-articular or para-articular erosions bounded by sclerotic borders can be identified, as well as osteophytes partially covering the tophi. The joint space is well preserved up to the advanced stage of the disease. No osteopenia is observed, which is common in other forms of arthritis. ${ }^{6}$ The increase in uric acid level may also contribute to the onset of inflammatory systemic comorbidities, and be associated with more severe cases of psoriasis. Gisondi et al. compared uric acid levels of individuals with psoriasis and control groups matched by age, sex, and body mass index. They found significantly higher values in patients with

\section{REFERENCES}

1. 1. Voiculescu VM, Lupu M, Papagheorghe L, Giurcaneanu C, Micu E. Psoriasis and Metabolic Syndrome--scientific evidence and therapeutic implications. J Med Life. 2014;7:468-71.

2. Santos M, Fonseca HM, Jalkh AP, Gomes GP, Cavalcante Ade S. Obesity and dyslipidemia in patients with psoriasis treated at a dermatologic clinic in Manaus. An Bras Dermatol. 2013;88:913-6.

3. Liu M, Li JH, Li B, He CD, Xiao T, Chen HD. Coexisting gout, erythrodermic psoriasis and psoriatic arthritis. Eur J Dermatol. 2009;19:184-5.

4. Punzi L, Scanu A, Ramonda R, Oliviero F. Gout as autoinflammatory disease: new mechanisms for more appropriated treatment targets. Autoimmun Rev. 2012;12:66-71.

5. Chandra A, Ray A, Senapati S, Chatterjee R. Genetic and epigenetic basis of psoriasis pathogenesis. Mol Immunol. 2015:64:313-23.

6. Kuo CF, Grainge MJ, Mallen C, Zhang W, Doherty M. Comorbidities in patients with gout prior to and following diagnosis: case-control study. Ann Rheum Dis. 2016;75:210-7

7. Gisondi P, Targher G, Cagalli A, Girolomoni G. Hyperuricemia in patients with chronic plaque psoriasis. J Am Acad Dermatol. 2014;70:127-30.

8. Kwon HH, Kwon IH, Choi JW, Youn Jl. Cross-sectional study on the correlation of serum uric acid with disease severity in Korean patients with psoriasis. Clin Exp Dermatol. 2011;36:473-8. psoriasis. By logistic regression analysis, the authors also concluded that psoriasis was the strongest predictor of hyperuricemia in the studied population, after adjustment for age, sex, and components of the metabolic syndrome. ${ }^{7}$ In another study, Kown et al. correlated hyperuricemia with a greater severity of the disease and with higher PASI (Psoriasis Area Severity Index) scores. ${ }^{8}$ In 2011, Isha et al. investigated the levels of uric acid in 24 patients before and 12 weeks after the treatment of psoriasis. They demonstrated statistically significant decreased levels of uric acid in the blood of patients with improvement in psoriasis clinical picture. ${ }^{9}$ The association of psoriasis with hyperuricemia can trigger the onset of gouty arthritis and disabling pain, and pose a greater risk of developing other inflammatory comorbidities, especially cardiovascular. Thus, periodic investigation of uric acid levels and consequent early treatment of alterations triggered by hyperuricemia are essential. ${ }^{10}$

\author{
MAILING ADDRESS: \\ Mônica Santos \\ Rua Codajás, 24 \\ Cachoeirinha \\ 69065-130 -Manaus, AM \\ Brazil \\ E-mail:m.n.souza.santos@gmail.com
}

How to cite this article: Lobato LC, Coutinho JC, Frota MZM, Schettini APM, Santos M. Chronic tophaceous gout in patients with psoriasis. An Bras Dermatol. 2017;92(1):104-6. 